\title{
Estetica e semiotica: il "ribaltone" post-strutturalista*
}

\section{Preliminari}

Questi preliminari riguardano precisazioni necessarie circa i termini "estetica", "ribaltone" e "semiotica" disseminati a vario titolo nell'argomento di questa mia conversazione.

Intanto quello di "estetica". Sappiamo tutti che i termini significano ciò che la storia, e nella storia volta a volta le diverse culture, fanno loro significare. Sappiamo che non sono portatori (come anche Popper tra altri - tra numerosi altri - ha sottolineato con efficacia) di alcun significato immobile, metafisico. Fuor di ogni posizione indebitamente dogmatica sappiamo che anche il ricorso al loro etimo, che a volte ci accade di fare (e non escludo che mi accada anche nel corso di questa mia conversazione), non intende riportarli al loro significato più vero e assoluto, ma solo recuperarli in un loro significato relativo, nel caso appunto alle esigenze della situazione che li ha visti nascere, considerato, nella nuova situazione, analogamente opportuno e funzionale. Non altro. Anche il termine "estetica" non è da meno. Nella sua storia ha finito per significare tutto e il contrario di tutto: l'esperienza, l'esperienza al suo livello sensibile, la sensibilità ridotta all'arte e così via e così via ... . Il vivere, insomma, a qualche suo livello, ma anche e appunto la sua assenza, il suo contrario: la semplice sua presa di coscienza, la sua semplice 'scienza'. Ed è in questa accezione in senso stretto che qui sarà inteso. Accezione galileiana e, per altro, già presente nello stesso Baumgarten (non si dimentichi: Aesthetica est scientia) qui ovviamente depurata, alla luce di tutta la più credibile riflessione epistemologica novecentesca, di ogni portato ontologico, positivista e neopositivista che sia.

Quindi il termine "ribaltone", novello neologismo di grande corso nell'attuale, travagliata, vita politica italiana, dove si caratterizza per due marche semantiche prevalenti e costanti: rovesciamento e tradimento. Ovunque e comunque: tanto se il termine viene usato da quella parte politica che si ritiene abbandonata per definire gli ex-alleati, quanto se viene usato da questi ultimi nei confronti di coloro che hanno abbandonato. Rovesciamenti e tradimenti voluti come reciproci e reciprocamente, per altro, negati. Ė chiaro che non è luogo, qui, per entrare in queste spinose e deprimenti questioni politiche nostrane. Mi interessa, invece, il possibile uso metaforico di questo termine per descrivere quanto succede, in campo estetologico, tra strutturalismo e post-strutturalismo. Tale termine mi sembra più 
che appropriato, non solo per le sue denotazioni, ma anche per i disagi connotativi cui si associa. Anche qui assistiamo a un rovesciamento e, mi pare, a un tradimento, ma non di una precedente verità, bensì a sua volta (come già ci si rimprovera là, in campo politico) di un altro suo tradimento e rovesciamento. Con effetti, anche qui, ripeto, di penose futilità.

Con il termine "semiotica" poi, e in fine, si rinvia semplicemente al campo in cui queste posizioni sono venute formandosi e, insieme, allo sfondo teorico che ha dato loro vita e figura. Campo indicato, qui, al suo livello semplicemente intuitivo e spontaneamente condiviso. Se saranno necessarie puntualizzazioni e precisazioni le farò strada facendo. Ma andiamo con ordine.

Che cosa avrebbero tradito le reciprocamente rovesciate posizioni dello strutturalismo e del post-strutturalismo? Lo dico subito, senza sfumature e vie traverse. Hanno tradito, a mio parere, l'esplicitazione convincente, la descrizione corretta, di quella che a me pare la struttura logicamente costitutiva dell'arte, la presa di coscienza convincente del principio insomma d'artisticità. Prima, criticamente, in generale, dove tale principio si confonde con il principio epistemologico della costituzione dell'identità di qualsiasi entità, naturale o artefatta che sia, che entri in rapporto con la nostra cultura, con i nostri modelli di coltivazione (il termine "cultura" va qui riportato ecco, appunto, al suo 'etimo') del mondo in generale e poi, inevitabilmente, nell'individuazione storica di quello che dovrebbe essere proprio dell'arte nella nostra attuale cultura occidentale o orientale che sia, nelle misura in cui anche quella cultura si va sempre di più occidentalizzando. Che poi la struttura vada separata dai movimenti scientifici che se ne occupano mi pare intuitivo. Pensarla diversamente sarebbe come pensare che il DNA, per esempio, sia un'invenzione della biologia e non della vita stessa. La struttura è propria del vivente (naturale e culturale) e buttarla via con i movimenti che ne falliscono la descrizione sarebbe ancora come buttare via il bambino con l'acqua sporca. Cosa letale per una scienza (e tutti questi movimenti vogliono, implicitamente o meno, essere scienza, dire cioè 'le cose' come stanno) che non rinunci a dirsi correttamente tale.

Che cosa occorrerebbe pensare, allora, per procedere scientificamente in questa ricerca? Ecco, io continuerei in questo modo. Indicherei, innanzitutto, che cosa sarebbe stato opportuno vedere, che cosa si sarebbe 'dovuto' vedere, in proposito, per servirmi poi di questo 'veduto', di questa 'visura' del reale - per dirla con un termine più à la page —, al fine d'individuare gli errori dei due movimenti in questione.

Certo, sto schematizzando molto e molto irrigidendo, ma, essendo le questioni complessissime e lo spazio poco, confido molto nei miei gentili lettori e nelle loro capacità correttamente ricostruttive del mio discorso. Del resto, volendo fare un quadro anche al minimo esauriente del problema, non si può proprio fare altro.

Cominciamo, allora, con ciò che si sarebbe dovuto, a mio parere, vedere. Farò a tal fine ricorso ad apologhi, a parabole, a microracconti insomma e a immagini. 
Mi danno icasticamente ciò che teoricamente richiederebbe lunghi giri di parole. Ma, come tutte le esemplificazioni animate, anche queste possono potenzialmente portare nella trattazione elementi devianti e spuri. So che i miei lettori saranno capaci di raccogliere da essi quanto logicamente serve a capirmi e che lasceranno cadere tutto il resto. Quindi, niente paura a procedere in tal modo.

\section{La potenza costitutiva dei luoghi}

Prendiamo la domanda "Mi dà un caffê?" È un esempio cui ricorro spesso per dire quanto, in proposito, voglio dire e ad essa, anche qui, mi affiderei.

Supponiamo che un alieno arrivi sul nostro pianeta e che, impossessatosi d'acchito (non si vede infatti perché, in lui, non dovrebbe essere finalmente attiva allo stato puro quella prensile fantasia catalettica, che invece a noi, malgrado $i$ noti auspici degli stoici, fa ancora tanto difetto) dell'insieme del nostro lessico e di tutte le sue regole per usarlo, voglia subito formulare, anche lui, la domanda corrente capace di fargli assaggiare quella strana cosa che noi chiamiamo appunto caffè. Gli basterà, per ottenerlo, operare sull' 'indeterminato' (sull'entropico) campo del lessico e delle sue regole combinatorie la doppia selezione (la doppia scelta, la doppia 'determinazione' o messa a fuoco che dir si voglia) delle cinque fonie - quattro le parole e una, l'intonazione interrogativa - da un lato, e della regola (anch'essa tra le tante) della loro unione (nel caso: pronome + verbo + articolo, ecc.)? Evidentemente no e credo che siamo tutti d'accordo. Sarà infatti necessario che tale messa a punto (messa in determinazione) del linguaggio, al fine di ottenere codesto benedetto caffè, avvenga all'interno di un bar o, se volete concedo - in qualsiasi altro luogo, ma non, per esempio, da un palcoscenico, all'interno di una recita. Ve l'immaginate la sorpresa del nostro alieno, nel caso avesse a chiedere a qualcuno del pubblico un caffè da tale luogo? Nessuno si alzerebbe per portarglielo e anche se un barman, casualmente presente in sala, avesse (sovrappensiero facciamo, per condizionamenti alla Pavlov, tanto per intenderci) ad alzarsi e a portarglielo, nessuno (cosa ancora più choccante per il nostro alieno) riuscirebbe a vivere come reale tale evento. Se poi il nostro alieno avesse anche a berlo, questo caffè, nessuno (colmo della beffa) penserebbe che egli ha realmente bevuto un caffe, ma soltanto che egli ha recitato (come dire?) la 'bevuta' di un caffè e nulla più. Con sua grande sorpresa, il nostro alieno si accorgerebbe che per possedere una lingua non basta possederne il lessico e il codice combinatorio (grammatica e sintassi), ma occorre possederne anche le convenzioni (i meta-codici, diciamo così) d'uso: questi soltanto capaci di far lingua una lingua, tanto in senso non-referenziale che referenziale (comunicativo, in senso stretto).

Non c'è linguaggio senza esecuzione sintattico-grammaticale del lessico. $\mathrm{Ha}$ ragione $\mathrm{H}$. Weinrich: solo l'esecuzione sintattico-grammaticale fa passare il significante dei termini dall' 'ampio', dal 'vago', dal 'sociale', dall' 'astratto', in una parola, dall' indeterminato' al 'determinante' della 'circoscrizione', della 
'precisione', dell' 'individuazione' e della 'concretezza', ma è soltanto l'a priori delle istituzioni, dei luoghi, dentro i quali tale esecuzione avviene, che ci dice che cosa dobbiamo poi farne di tale esecuzione determinata, in che senso dobbiamo poi usarla.

Luoghi (istituzioni) raggruppabili, per noi - oggi - , in almeno due grandi classi logiche: quelli (tutti) dell'arte e quelli (tutti) non dell'arte. Teatro, appunto, nella prima classe, ma anche (che so) galleria d'arte, collane di poesia, di narrativa ecc. ecc., vicariabili, si capisce, da una loro semplice indicazione semiosica, magari sotto forma di titolo apposto all'opera stessa. Per esempio quello di "poesia", scritto su qualcosa che si vorrebbe venisse fatto vivere come poesia e così via. Ogni altro luogo non deputato a mostrare arte, invece, nella seconda.

Nel caso della frase eseguita al bar, convenzione vuole (e siamo tutti d'accordo, penso) che si debba fare attenzione solo e soltanto al concetto che essa intende veicolare. O che forse, al fine di aver un caffe al bar, conta anche la nostra voce, chioccia, rauca o solenne che sia? No: tutta roba, questa, non pertinente alla circostanza in questione. Nel caso della sua esecuzione teatrale, invece, convenzione vuole che proprio codesto suo aspetto, codesto suo appiattimento comunicativo a livello concettuale non abbia più alcun senso e che tutto, nella frase, diventi, se trova paradigmi capaci di leggerlo, significativo. Tazzina di caffê? Perché non nero abîme di insospettate concrescenze psicoanalitiche, tanto più insondate (si sa) quanto più quotidiane? Perché non simbolo, ancora, di indicativissime ritualità antropologiche e sociopolitiche e così via? A convincerci - ha ragione Roland Barthes, seppure con le dovute integrazioni - basterebbe la loro semplice organizzazione discorsiva in interna coerenza (44). Non altro. Non è che senza l'attenzione ai luoghi non ci sia l'attenzione al livello pragmatico del linguaggio e dell'uso in generale dei segni: non c's proprio il linguaggio. C'è solo materia ancora indeterminata (costrutti inerti) da significare. Costrutti che diverranno poesia (arte) se praticati secondo la convenzione, la langue (la 'struttura' d'uso, appunto) dell'arte (dei luoghi dell'arte) e semplice comunicazione strumentale, invece, se praticati secondo quella dei comuni luoghi discorsivi: bar, l'aula in cui è stata letta questa conferenza, ecc. ecc.

Non può essere qualcosa ad essa intrinseco a spiegarci l'approdo della frase indicata ("Mi dà un caffê?") alle due, e opposte, identità culturali appena ipotizzate. La frase, infatti, rimane materialmente la stessa. La causa di questo suo mutare di identità non potrà che essere funzione di ciò che, nell'esperimento, cambia e cioè della sua circostanza d'uso, del luogo insomma, in cui si dà la sua esecuzione. Così per lo Scolabottiglie di Duchamp e per gli infiniti ready-made dell'arte contemporanea. Non è qualcosa di strutturalmente ad essi intrinseco a spiegarci la loro differenza da un normale oggetto d'uso. Duchamp non ha fatto alcun intervento sulla struttura materiale dello scolabottiglia, sulla sua forma. Il suo Scolabottiglie è rimasto percettivamente indiscernibile da ogni altro. Cos'è invece cambiato nell'operazione di Duchamp? Soltanto il luogo dove lo scolabottiglie è stato posto: la galleria d'arte al posto di un normale negozio da canti- 
niere o cantina che sia. E qui, nel luogo, sta la sua raison d'être arte. Non in altro. Del resto che cosa può permettere, per esempio, ai Promessi sposidi Alessandro Manzoni e alla Merda d'artista di Piero Manzoni di stare insieme nel campo dell'arte? Che poi stiano insieme in questo campo non ci sono dubbi: vive nelle biblioteche di letteratura, la prima opera, e nei musei la seconda. Non certo qualche tratto materialmente comune. E se non è che qualche tratto materiale ad esse intrinseco, non potrà che essere un qualche tratto 'funzionale' ad esse esterno. Ed è questa 'struttura funzionale' esterna agli oggetti e alla cose che bisogna cercare per capire il principio costitutivo della loro culturale identità, nel caso quella propria dell'arte.

Qualcuno potrebbe obiettarmi che tutto ciò va bene soltanto per quella che diciamo arte concettuale, ma non per l'arte in generale. Ebbene, io credo, invece, che sia vero il contrario. Credo che nell'arte cosiddetta concettuale venga a nudo, finalmente, un principio d'artisticità che non è solo suo, ma proprio dell'arte in generale.

La Gioconda non è arte in sé, ma per la cultura e solo per quella che l'ha delegata a funzionare come tale: con essa destinata a vivere e morire. La catena di oblii e di riscoperte di cui è fatta la storia dell'arte, le liti sui restauri o meno e, soprattutto, l'insensibilità di culture 'radicalmente' diverse dalla nostra, prive della nostra nozione di arte, verso questi valori insegnano o, meglio, dovrebbero insegnarci molte cose al riguardo. Del resto questo principio, come qua e là già mi sono lasciato andare ad affermare, pare proprio vero in generale. Già Platone ci ammoniva. Non si dimentichi: chi ha l'arte di fare le selle? Guai a rispondere il sellaio. Chi ha l'arte di fare i flauti? Guai a rispondere il costruttore di flauti. È il cavaliere, e cioè chi la usa, che ha l'arte di fare la sella e se il sellaio la sa fare è perché non è privo del sapere del cavaliere. Così per il flauto e chi lo suona e chi lo fa. "Cavalcare": una pratica a soggetto diffuso (tutti possono cavalcare) come appunto quelle che indicano i nostri luoghi: al bar e in una galleria d'arte non uno solo ma tutti possiamo entrare. Guai a dimenticare (a tradire) i luoghi, a non tenerli, insomma, vigilmente presso di sé. Ne vengono, ripeto, guai gravissimi: quelli, appunto, di cui ritengo siano teoreticamente responsabili i due movimenti in questione, che si muovono sì per posizioni rovesciate, ma entrambi tradendo questo loro dovere teoretico, questo compito teoreticamente corretto che essi stessi, per altro, si sono dato e si danno. Vediamo.

\section{Il luogo tradito}

Mi servirei, per organizzare quanto penso al riguardo, di un precedente tentativo d'organizzazione della stessa materia da parte del mio collega d'università e di corso Umberto Eco.

Umberto Eco, postosi anche lui, non molto tempo fa, di fronte al problema del luogo dove cercare il principio costitutivo dell'artisticità e dei conseguenti comportamenti della critica (delle conseguenti legalità che la critica arriva a ricono- 
scere a se stessa) nei confronti dell'opera, ha creduto di poterne perimetrare il campo secondo le seguenti tre intenzioni: 'intentio' auctoris, 'intentio' operis e 'intentio' lectoris ("Appunti"). Si muova pur fin che vuole, questo principio, la storia lo muova pure fin che crede, i confini del suo moto non cambieranno: questi sono e questi resteranno, da essi non si può uscire. Ma andiamo con ordine. Occupiamoci prima di tutto dello strutturalismo. Cosa possiamo dire di esso a partire da questa cartina di tornasole propostaci da Eco?

Del cosiddetto strutturalismo, perché poi sappiamo quanto disagio tale etichetta ha creato a quelli che sono stati considerati i suoi adepti. Ma, insomma, il movimento, malgrado loro, l'ha avuto un volto comune o, meglio, è stato voluto da chi ad esso s'è 'istituzionalmente' ispirato (ed è questo che qui conta, se dello strutturalismo dobbiamo occuparci) con un volto comune e a questo volto facciamo qui riferimento.

Volto che, andando alla sua origine, vedrei delineato in quello che ne può essere considerato il padre fondatore, intendo $\mathrm{R}$. Jakobson e la sua teoria delle funzioni dell'atto linguistico, che ben sappiamo quanto ha influenzato non solo la riflessione specialistica sull'arte, ma anche e più estesamente la cultura in generale e la scuola soprattutto.

Ebbene. Dove pone Jakobson il principio d'artisticità? Di che cos'è funzione l'artisticità per lui? Non forse di una lavorazione anomala (inusuale) del messaggio ad opera dell'emittente? Lavorazione che comincerebbe a dare i suoi frutti (l'arte appunto) quando arrivasse ad avere, come ancora Eco, trascrivendo Jakobson in termini semiotici, conferma, una confezione tipo: "le idee verdi senza colore" - frase, questa, ormai mitica - "dormono furiosamente" (Eco, "Il testo estetico"). E non è questo come dire che l'artisticità sarebbe effetto di un testo voluto (mi perdonino i miei gentili lettori, ma comprendano che sono sempre più costretto a procedere così, alla grossa) intrinsicamente anomalo dal suo stesso autore e quindi che luogo costitutivo dell'artisticità sarebbe, diciamo, il blocco in solido dell' 'intentio' operis e dell' intentio' auctoris? Non osta poi affatto a questa congettura la constatazione che tutto questo lavorio di confezione dovrebbe sì oscurare il testo, ma non fino al punto da togliere ad esso l'intenzione comunicativa (monosemica nei miei termini) referenziale. Anzi, la corrobora a mio parere definitivamente. Un'intenzione che il testo può solo ambiguare e non togliere di mezzo è necessariamente un'intenzione che lo precede e quindi un'intenzione non del testo ma del suo autore. Ė il plesso intentio operis-intentio auctoris, allora, che nello strutturalismo viene in primo piano, senza eliminare il lettore (il fruitore) si capisce. Il riferimento (la referenza), per quanto ambiguo (ambigua), deve pur passare e passare per chi se non per un suo ricevente? Il tutto però secondo lo schema della comunicazione corrente, dove l' intentio' lectoris non ha alcuna autonomia, ma sta, se sta, al servizio dell'emittente e solo di quello. La semiosi dell'arte non avrebbe statuto diverso, per lo strutturalismo, da quello della comunicazione strumentale in senso stretto. Sarebbe la stessa, solo un po' più complicata. Un po' come accadeva ai tolemaici di pensare: i movi- 
menti di Marte non negavano, secondo loro, la regola del circolo, la rendevano soltanto un po' più complicata.

Che fa, per altro, il post-strutturalismo? Vale la perimetrazione delle tre intenzioni di Eco anche per dare ragione di quanto esso afferma? Beh! . . . in linea di massima direi proprio di sì. Dove porre infatti il discrimine per separarlo dallo strutturalismo in senso stretto? Credo proprio si possa dire nel fatto che il poststrutturalismo non manca di riconoscere l'avvenuto potenziamento, da parte della nostra cultura, dell'intenzione del fruitore a scapito di quella dell'autore. Non dell'opera, si capisce, ma di quello dell'autore sì. E allora a un plesso, al plesso intentio operis-intentio auctoris se ne sostituirebbe, per il post-strutturalismo, un altro: quello opposto dell'intentio lectoris-intentio operis, semplicemente.

Movimento questo, del cosiddetto post-strutturalismo, difficile da indicare nei suoi insiemi precisi e nei suoi autori. Si potrebbe in senso lato dire orizzonte dell'ermeneutica, del decostruzionismo forse, di ogni convenzionalismo, ecc. Ma con grande cautela, trattandosi a volte, e ancora, di strutturalismo furbastro, di strutturalismo mascherato quando non addirittura di vaghezze, di allucinazioni, si potrebbe dire alla Kant, che tutto vedono (tutto quanto desiderano vedere) tranne ciò che occorrerebbe proprio criticamente (scientificamente) vedere. Ma il discorso andrebbe fatto posizione per posizione, autore per autore. Cosa che qui ovviamente occorre lasciar perdere. Lo spazio non c'è più e il problema è complessissimo. Accontentiamoci del discrimine. Se siamo d'accordo sul discrimine, per procedere non serve altro.

Bene. Il rovesciamento (siamo di nuovo al ribaltone) è evidente. Abbiamo: plesso opera-autore, da una parte (strutturalismo), e fruitore-opera dall'altra (post-strutturalismo). Ma il tradimento dei 'luoghi' resta comune. Come ragionarne, pretendendone 1'attenzione? Vediamo. Lo farei, anche qui, allo stesso modo e per la stessa via che, a suo tempo, seguii per difenderne la necessità occupandomi della perimetrazione di Eco (Nanni). Vediamo.

\section{La questione della barca, a conclusione}

Proporre le tre intenzioni indicate come perimetro sufficiente a dare ragione del costituirsi dell'artisticità di una qualche entità e dei suoi movimenti, sarebbe come pensare, dissi allora e ripeto ora, di potere dare ragione dell'identità di "barca" di una barca, ricorrendo unicamente all'intenzione del suo costruttore, della barca stessa (se così si può dire) e a quella del barcaiolo che la usa, dimenticandone un'altra ben più importante e profonda, non quarta rispetto ad esse, ma prima, perché loro matrice appunto profonda. Intenzione di cui quelle tre sono semplicemente la fenomenologia di superficie, pronte a svanire alla sua scomparsa, come scompare la neve al sole. Intendo l'intenzione (la logica) del mare. Solo il mare, solo il 'luogo-mare' e la sua logica possono darci esauriente ragione dell'essere della barca, del suo costruttore, del barcaiolo oltre che dei loro rapporti. Non altro. Intenzione, questa, che in ambito culturale ho proposto di chia- 
mare intentio culturae e che ha nei luoghi collettivi (bar, appunto, galleria d'arte, ecc.) i suoi fisici significanti.

Lo strutturalismo non sa nemmeno cosa siano questi luoghi, ma a dir il vero il post-strutturalismo comincia ad accorgersene. Penso, per esempio, a S. Fish. Penso ad A. Danto. Penso, in Italia, a G. Vattimo anche e così via. Anche se poi non ne sanno trarre le dovute conclusioni. Hanno la soluzione in mano e se la lasciano sfuggire. Fish, credendo che oltre l'intuizione del loro decisivo potere, non ci sia altro poi da vedere e da dire o forse non accorgendosi proprio di quanto gli accade di intuire e di vedere. Danto, finendo per optare, nel rispondere al quesito posto da questi luoghi, per un aristotelismo di maniera che confonde l'effetto con la causa. Vattimo, perché ha altri interessi a cui pensare, più etico-politici che scientifici tout court.

Abbiamo, per altro, intravisto all'inizio che non solo tali luoghi sono attivi, ma anche che, da questo punto di vista, si suddividono secondo due diverse logiche d'uso dei costrutti semiosici e delle cose: quella pratica e quella diversa propria dell'arte. ${ }^{1}$ A me pare che il problema più cogente dell'estetologia scientifica contemporanea dovrebbe essere quello di tentare di portare a descrizione questa langue propria della nostra arte, oggi. È questo che nei miei ultimi vent'anni di lavoro, dandone volta a volta i risultati nei miei ultimi quattro libri, ho tentato, contro lo strutturalismo, di fare. Naturale che in ciò m'aspettassi qualcosa, qualche collaborazione dalla ventata post-strutturalista. Ma per ora, più che mettersi - con qualche suo rappresentante - su questa mia strada, altro non pare saper fare. Dice Culler: "Per Frye, naturalmente, l'alternativa sta in una poetica che tenti di descrivere le convenzioni e le strategie con cui le opere raggiungono i loro effetti" (141). Benissimo. È questo che si deve fare, purché siano però poetiche (langue) d'uso e non di produzione. Ha ragione F. de Saussure: spesso è piu facile vedere una verità che metterla al posto giusto.

\section{Università di Bologna}

\section{NOTE}

* Conferenza tenuta all' Università di Toronto il 3 aprile 1995.

l Nel caso Eco (e compagnia strutturalista) desse (dessero) per scontata 'l'intenzione' del luogo e quindi ritenesse (ritenessero), per questo, inutile occuparsene, io avrei ancora più ragione a sostenere che essi non ne vedono la potenza originariamente costitutiva e non s'accorgono di quanto questa potenza cambi passando dai luoghi discorsivi in senso stretto a quelli dell'arte. Se io, telefonando a mia moglie, non sto ogni volta a precisare che le telefono dalla Terra è perché do per scontato di non poter essere in nessun altro luogo. Ma se mi si desse la possibilità di poter essere tranquillamente anche su Marte, credo la precisazione suddetta non la tacerei, dando insieme testimonianza della mia consapevolezza circa l'importanza dei luoghi e della loro radicale diversità. Diversità che i nostri strutturalisti non dimostrano, con il loro silenzio, di aver coscienzializzato. Sia che tacciano, perché refrattari in assoluto all'importanza dei luoghi, sia che tacciano convinti che il luogo del linguaggio, pur attivo, sia però uno e uno solo (quello del bar) la cosa per noi non cambia: 
l'autonomia dell'arte non viene vista e la logica dei suoi luoghi ideologicamente ridotta a quella dei luoghi del discorso comune.

\section{BIBLIOGRAFIA}

Barthes, Roland. Critica e verità. Torino: Einaudi, 1966.

Culler, Jonathan D. "In difesa della sovra-interpretazione".. Interpretazione e sovrainterpretazione. Ed. Umberto Eco. Milano: Bompiani, 1995.

Danto, Arthur C. La destituzione filosofica dell' arte. Tema celeste, 1992.

Eco, Umberto. "Appunti sulla semiotica della ricezione". Carte semiotiche 2 (1986): "Il testo estetico come esempio di invenzione". Trallato di semiotica generale.

Milano: Bompiani, 1975.

Fish, Stanley E. C' è un testo in questa classe? Torino: Einaudi, 1987.

Jakobson, Roman. "Linguistica e poetica". Saggi di linguistica generale. Milano: Feltrinelli, 1974.

Nanni, Luciano. "Le legalità della critica". I cosmi, il metodo (diario d'arte e di epistemologia). Bologna: Book Editore, 1994.

Vattimo, Gianni. Oltre l'interpretazıone. Bari: Laterza, 1994.

Weinrich, Harald. Metafora e menzogna: la serenità dell' arte. Bologna: Il Mulino, 1976. 